\title{
A four particle velocity sensor device
}

\author{
D. R. Yntema, W. F. Druyvesteyn, ${ }^{\text {a) }}$ and M. Elwenspoek \\ Division of EEMCS, University of Twente, P.O. Box 217, 7500 AE Enschede, The Netherlands
}

(Received 21 February 2005; revised 23 September 2005; accepted 21 November 2005)

\begin{abstract}
In a sound field disturbance of pressure, particle velocity, density, temperature, and energy occur. In this paper acoustic disturbances in air are considered. In the majority of papers on acoustics only changes in the sound pressure are reported while in this paper results on the particle velocity are reported. Since particle velocity is a vector, while the pressure is a scalar, more information can be obtained when using a particle velocity sensor instead of a pressure sensor (microphone). Four particle velocity sensors are combined to one (small) device. In a reverberant room the four autospectra and the six cross spectra are determined. Interpretation of the measured results gives information of the free field (sound field without a contribution of reflections) as well as of the reverberant field. (C) 2006 Acoustical Society of America. [DOI: 10.1121/1.2151797]
\end{abstract}

PACS number(s): 43.58.Fm, 43.55.Mc [AJZi]

Pages: 943-951

\section{INTRODUCTION}

In an acoustic disturbance in air, which is considered in this paper, (small) variations of the pressure $p$, the particle velocity $v$, the density $\rho$, or the temperature $T$, occur. Sensors for measuring the particle velocity in water are widely known, see Ref. 1. In air, often two closely spaced $p$ sensors are used, from which the gradient of the pressure is estimated, which via the force equation is directly related to the particle velocity. Also a particle velocity sensor based on ultrasonic transduction has been proposed. ${ }^{2}$ Two parallel ultrasonic beams are launched in opposite directions. The time difference in traveling time of the ultrasonic sound waves is proportional to the particle velocity. Nowadays a $v$ sensor, the Microflown or $\mu$ flown, ${ }^{3}$ is available and the particle velocity can be measured directly. Various experiments ${ }^{3}$ have been performed to prove that with this sensor indeed the particle velocity is measured. Recently, ${ }^{4}$ a direct comparison has been made by measuring the particle velocity at the same position with the method of Laser Doppler Anemometry (LDA), with the $\mu$ flown, a good agreement was found.

\section{ONE V SENSOR}

As a sensor for the particle velocity we used the "Microflown or $\mu$ flown." "3 This sensor consists basically of two thin wires, parallel to each other, which are heated up to about $200-300{ }^{\circ} \mathrm{C}$ by an electrical current; see Fig. 1 . The temperature profile around the wires is influenced by the particle velocity of an acoustic disturbance, resulting in a small resistance change of the wires. In fact, the sensor detects the particle velocity component in a direction perpendicular to the length of the wires and in the plane of the wires. Define a unit vector $\boldsymbol{\mu}$, perpendicular to the length of the wires and in the plane of the wires, then the electrical signal of the sensor is proportional to the inner scalar product $\boldsymbol{\mu}$.v. Throughout this paper we denote this unit vector as the sensitivity unit vector of the sensor. We often take the propor-

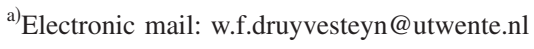

tionality constant equal to one and write for the sensor signal $s(t): s(t)=\boldsymbol{\mu} \cdot \mathbf{v}(t)$. The directional characteristic of this $v$ sensor is independent of the frequency and follows from this relation as: $\boldsymbol{\mu} \cdot \mathbf{v}=|\mathbf{v}| \cdot \cos \left(\alpha_{\boldsymbol{\mu v}}\right)$, where $\alpha_{\mu v}$ is the angle between $\mathbf{v}$ and $\boldsymbol{\mu}$. A detailed model is presented in Ref. 5 .

Since the measurement of the particle velocity is directly derived from the measurement of the electrical resistance of the sensor, the "Johnson" or resistance noise forms a lower limit of the noise of the microflown: $\left\langle V_{\text {noise }}^{2}\right\rangle_{f, f+\Delta f}$ $=(4 \mathrm{kTR}) \Delta f$, where $k$ is the Boltzmann-constant, $R$ the resistance, $T$ the absolute temperature, and $\Delta f$ the frequency interval or bandwidth. The noise can be higher than this lower limit, if a less optimal amplifier is used. Additionally the $1 / f$ noise $^{6}$ is higher at low frequency than the resistance noise. In our experiments, ${ }^{6}$ the noise level above about $600 \mathrm{~Hz}$ was indeed practically equal to the Johnson noise. At lower frequencies the $1 / f$ noise dominates, see Fig. 2 and Ref. 6, the theoretical white noise level of a similar resistor is $-165 \mathrm{dBV} / \sqrt{ } \mathrm{Hz}$.

A direct comparison of the noise or signal-to-noise ratio (SNR) between a $v$ sensor and a $p$ sensor can be done via the concept of the selfnoise. The selfnoise of a sensor is the ratio of the generated noise in volt and the sensitivity in volt $/(\mathrm{m} /$ sec) for a $v$ sensor or volt/(Pa) for a $p$ sensor; the obtained values represent the acoustical selfnoise of the device. Using the far field relation $v=p / \rho c$, with $\rho$ the density of the air and $c$ the sound velocity, the selfnoise times $\rho c$ of the $v$ sensor can be compared with the selfnoise of the $p$ sensor.

The state of the art is that at low frequencies the selfnoise times $\rho c$ of the $v$ sensor is lower than the selfnoise of available $p$ sensors, while at higher frequencies the selfnoise of the $p$ sensor is lower. ${ }^{7}$

Consider now the sensor signal. For stationary signals the rms value, or the autospectrum, is measured, the latter being: $A=(1 / T) \int_{0}^{T} s(t) s^{*}(t) d t$.

Suppose a sound source is present at the origin of a Cartesian coordinate system emitting plane waves, propagating in the $x$ direction and a $v$ sensor is placed on the $x$ axis at a distance much larger than the wavelength and dimensions of the source (far field situation). When the sensitivity unit 


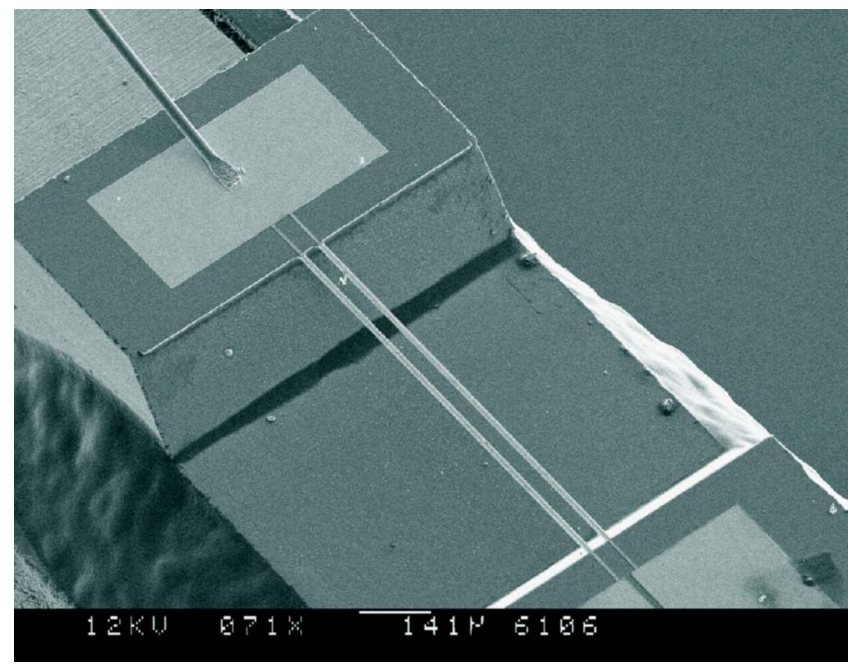

FIG. 1. The microflown sensor, SEM photo. The wires are both heating and sensing elements.

vector is along the $x$ axis, $\boldsymbol{\mu}=[1,0,0]$ the free field and a contribution of the reverberant field will be measured. If however, the sensor is rotated such that $\boldsymbol{\mu}=[0,1,0]$ or $\boldsymbol{\mu}$ $=[0,0,1]$ no contribution of the free field will be measured and only (a part) of the reverberant field is detected. In a pure diffuse reverberant sound field, where the distances from the mirror sources to the sensor are much larger than the wavelength and much larger than the dimensions of the sources, the relation between the rms value of the pressure and particle velocity is given by: $v_{\mathrm{rev}}^{2}=p_{\mathrm{rev}}^{2} /(\rho c)^{2}$. In a purely diffuse reverberant sound field the three components $v_{x}^{2}, v_{y}^{2}$, and $v_{z}^{2}$ are equal thus $v_{x}^{2}=v_{\text {rev }}^{2} / 3$ or $v_{x}^{2}=(1 / 3) p_{\text {rev }}^{2} /(\rho c)^{2}$; this relation will be discussed in more detail in Sec. III.

When the $v$ sensor is used for direct recording experiments with a sound source at the $x$ axis it is interesting to listen to the cases where $\boldsymbol{\mu}=[1,0,0]$ and $\boldsymbol{\mu}=[0,1,0]$. For the former case the direct and reverberant field is heard, while for the latter case one listens to only the reverberant field. It is also interesting to listen to the reverberant field in the room at different positions.

\section{TWO SENSORS}

In this section the response of two $v$ sensors for stationary signals is discussed; the orientation of the two $v$ sensors is given by $\boldsymbol{\mu}_{1}$ and $\boldsymbol{\mu}_{2}$. Three spectra can now be determined, two autospectra and one cross spectrum, the latter being

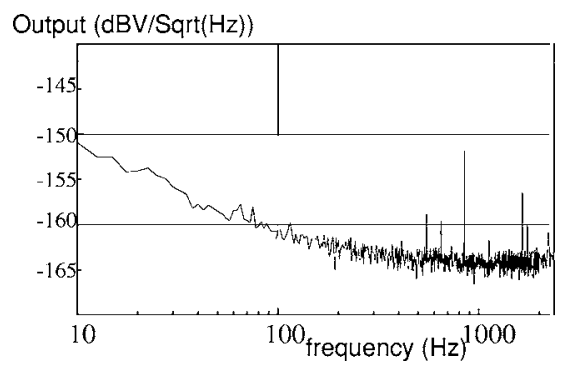

FIG. 2. Measured noise spectrum of a microflown.

$$
C_{i j}=(1 / T) \int_{0}^{T} s_{i}(t) s_{j}^{*}(t) d t
$$

The two autospectra $A_{i}$ and $A_{j}$ are found from Eq. (1) by taking $j=i$. Consider first the noise level of these signals. In most cases the noise level in the cross spectrum is lower than in the separate autospectra. The reason for this is that in the cross spectrum the product of the noise of sensor $i$ and the noise of sensor $j$ vanishes if the noise signals are uncorrelated and if the integration time $T$ is taken long (the same holds for the product of the signal times the noise). Gordienko et al. ${ }^{8}$ reported already in 1993 a reduction of the ocean noise field in the cross correlation of two sensors. Also, Shchurov ${ }^{9}$ reported an increase in the signal-to-noise ratio (SNR) when an intensity probe is used. For isotropic (diffusive) noise even an increase in the SNR of $20-30 \mathrm{~dB}$ was found. For the case of two microflowns a reduction of the noise, down to $30 \mathrm{~dB}$ for frequencies above $600 \mathrm{~Hz}$, has been reported. ${ }^{6}$ The reason for this enormous reduction is that for frequencies above $600 \mathrm{~Hz}$ the dominant noise is the resistance noise (or Johnson noise). One expects that the resistance noise from one wire (resistance) powered by one battery is uncorrelated with the noise from another wire (resistance) powered by another battery. For lower frequencies $1 / f$ noise appears, resulting in a much smaller reduction of the noise in the cross correlation. ${ }^{6}$ The reason for this is that the decrease in the noise of the cross correlation spectrum is most effective for flat, broadband, frequency spectra. If a $1 / f$ shaped component is present, there is a correlation between subsequent sample points $x_{i}$ and $x_{j}$, while in a flat spectrum there is no correlation between points $x_{i}$ and $x_{j}(i \neq j)$ in the series of data points. ${ }^{6}$ Second another remarkable quality of the signal is that the influence of the reverberant sound field on the cross spectrum on one hand and the autospectra on the other hand is completely different. For the cross spectra the contribution of the reverberant field can even be zero, if $\boldsymbol{\mu}_{1} \cdot \boldsymbol{\mu}_{2}$ $=0$, as will be explained below.

A simple two-dimensional case is worked out first. Two $v$ sensors at the origin of the $x-y$ plane are present, with $\boldsymbol{\mu}_{1}=[1,0]$ and $\boldsymbol{\mu}_{2}=[0,1]$. The two-dimensional purely diffuse reverberant sound field is modeled as four mirror sources $M_{1 . .4}$ on a circle making an angle $\beta, \pi-\beta, \pi+\beta$, and $2 \pi-\beta$ with the $x$ axis. Consider the contribution to the product of $s_{1}(t)$ and $s_{2}(t)$ when for each mirror source the velocity is directed inward or outward. For source $M_{1}$ the product of $s_{1}(t) s_{2}(t)$ will be positive $\left[s_{1}(t)\right.$ and $s_{2}(t)$ are both negative for an inward velocity, since $\boldsymbol{\mu}_{1}=[1,0], v_{x}<0$ and $\boldsymbol{\mu}_{2}=[0,1]$, $v_{y}<0 ; s_{1}(t)$ and $s_{2}(t)$ are both positive for an outward velocity, since $\boldsymbol{\mu}_{1}=[1,0], v_{x}>0$ and $\boldsymbol{\mu}_{2}=[0,1], v_{y}>0$. So the product $s_{1}(t) s_{2}(t)$ is positive]. For source $M_{2}$ the product $s_{1}(t) s_{2}(t)$ is negative $\left[s_{1}(t)>0, s_{2}(t)<0\right.$ for an inward velocity and $s_{1}(t)<0, s_{2}(t)>0$ for an outward velocity], for $M_{3}$ the product is positive and for source $M_{4}$ again negative. So for all mirror sources, equally distributed over the circle and a long integration time, the total contribution will be zero.

Now consider the more general situation with a threedimensional pure diffuse reverberant sound field with two $v$ sensors in the origin with vectors $\boldsymbol{\mu}_{1}=\left[\mu_{1 x}, \mu_{1 y}, \mu_{1 z}\right]$ and 
$\boldsymbol{\mu}_{2}=\left[\mu_{2 x}, \mu_{2 y}, \mu_{2 z}\right]$. Define first $v_{\text {rev }}^{2}$. Suppose a hypothetical omnidirectional $v$ sensor is in the origin [this is certainly not a $\mu$ flown, which has a $\cos \left(\alpha_{\mu v}\right)$ dependence, see Sec. I]. The mirror sources are equally distributed over a sphere. An arbitrary mirror source makes an angle $\alpha$ with the $z$ axis and an angle $\beta$ in the $x-y$ plane, thus $v_{x} \propto \sin (\alpha) \cos (\beta), \quad v_{y}$ $\propto \sin (\alpha) \sin (\beta)$, and $v_{z} \propto \cos (\alpha)$. Take the $v_{\text {rms }}$ value from the sources within a solid angle $d \Omega=\sin (\alpha) d \alpha d \beta$, as $v_{m}$. The signal of the hypothetical omnidirectional sensor is proportional to $v_{\text {rev }}^{2}=\int v_{m}^{2} d \Omega=\int_{0}^{\pi} \sin (\alpha) d \alpha \int_{0}^{2 \pi} d \beta \cdot v_{m}^{2}=4 \pi v_{m}^{2}$. In the far field approximation $v_{\mathrm{rev}}^{2}=p_{\mathrm{rev}}^{2} /(\rho c)^{2}$.

The signal of the $v$ sensor with $\boldsymbol{\mu}_{1}$ is $s_{1}=\boldsymbol{\mu}_{1} \cdot \mathbf{v}_{\mathbf{m}}$ $=\mathrm{v}_{\mathrm{m}}\left\{\mu_{1 x} \cdot \sin (\alpha) \cos (\beta)+\mu_{1 y} \cdot \sin (\alpha) \sin (\beta)+\mu_{1 z} \cdot \cos (\alpha)\right\}$, thus the cross spectrum from the two $v$ sensors with $\boldsymbol{\mu}_{1}$ and $\boldsymbol{\mu}_{2}$ becomes

$$
\begin{aligned}
C_{12} & =\int_{0}^{\pi} \sin (\alpha) d \alpha \int_{0}^{2 \pi} d \beta\left(\boldsymbol{\mu}_{\mathbf{1}} \cdot \mathbf{v}_{\mathbf{m}}\right)\left(\boldsymbol{\mu}_{\mathbf{2}} \cdot \mathbf{v}_{\mathbf{m}}\right) \\
& =\nu_{\mathrm{rev}}^{2}\left(\boldsymbol{\mu}_{\mathbf{1}} \cdot \boldsymbol{\mu}_{\mathbf{2}}\right) / 3 .
\end{aligned}
$$

Equation (2) follows from simple straightforward algebra and is written out in Appendix A.

Equation (2) shows that if $\boldsymbol{\mu}_{\mathbf{1}}$ and $\boldsymbol{\mu}_{\boldsymbol{2}}$ are orthogonal to each other, there is no contribution of the reverberant sound field to the cross spectrum, and only the direct sound field of the source is measured. This is similar to the measurement of the real part of the sound intensity, for which also the reverberant sound field vanishes. ${ }^{10}$ If $\boldsymbol{\mu}_{\mathbf{1}}=\boldsymbol{\mu}_{\mathbf{2}}$ (two $v$ sensors in the same direction, in order to obtain a low noise level, as compared to the noise level in the autospectrum of one $v$ sensor) the contribution of the reverberant field is $v_{\text {rev }}^{2} / 3$. In Sec. IV the case of $\boldsymbol{\mu}_{1}=[1,1,0] / \sqrt{ } 2$ and $\boldsymbol{\mu}_{2}=[0,1,1] / \sqrt{ } 2$ is discussed; for this configuration the contribution of the reverberant field in the cross spectrum will be $v_{\mathrm{rev}}^{2} / 6$.
Thus when using two orthogonal oriented $v$ sensors it is possible in a reverberant room to determine the direct sound field ("free field measurements") of a source, as well as the reverberant sound field in the room.

\section{MORE V SENSORS}

When using two $v$ sensors it is possible that with a sound source in a room no direct sound is detected in $A_{1}, A_{2}$, and $C_{12}$. For example, if the sound source is at $[0,0,0]$ and the sensors at $[1,1,0]$ with $\boldsymbol{\mu}_{1}=[-1,1,0]$ and $\boldsymbol{\mu}_{2}=[0,0,1]$ no direct sound is detected: $A_{1}=A_{2}=v_{\mathrm{rev}}^{2} / 3$ and $C_{12}=0$. It seems logical then to use three $v$ sensors with $\boldsymbol{\mu}_{1}=[1,0,0], \boldsymbol{\mu}_{2}$ $=[0,1,0]$, and $\boldsymbol{\mu}_{3}=[0,0,1]$ and to use the three low-noise cross spectra for the source localization. However, for that case it may occur that all three cross spectra are zero; e.g., source at $[0,0,0]$ and sensors at $x$ axis (or $y$ axis or $z$ axis).

In order to anticipate this, four $v$ sensors should be used. In the device discussed below we used: $\boldsymbol{\mu}_{1}=[1,0,1] / \sqrt{ } 2$, $\boldsymbol{\mu}_{2}=[0,1,1] / \sqrt{ } 2, \boldsymbol{\mu}_{3}=[-1,0,1] / \sqrt{ } 2$, and $\boldsymbol{\mu}_{4}=[0,-1,1] / \sqrt{ } 2$. As measured spectra we now have four autospectra and six cross spectra. The contribution of the free field to the time averaged auto- and cross-spectra can be found as follows. Write the free field particle velocity vector and its time dependence as $\mathbf{v}\left(v_{x}, v_{y}, v_{z}\right) a(t)$, where the vector $\mathbf{v}\left(v_{x}, v_{y}, v_{z}\right)$, with (the real) components $v_{x}, v_{y}$, and $v_{z}$ represents the directional vector of the free field particle velocity and $a(t)$ the time dependence of the source signal. Write for the signal of sensor $A$, divided by the sensor sensitivity $[\mathrm{in} \mathrm{V} /(\mathrm{m} / \mathrm{sec})]$, the symbol $S_{A}(t)$, then $s_{A}(t)=\left(\boldsymbol{\mu}_{A} \cdot \mathbf{v}\right) a(t)=\left(\mu_{x A} \cdot v_{x}+\mu_{y A} \cdot v_{y}\right.$ $\left.+\mu_{z A} \cdot v_{z}\right) a(t) ; \boldsymbol{\mu}_{A}=\left[\mu_{x A}, \mu_{y A}, \mu_{z A}\right]$ is the sensitivity unit vector of sensor $A$. The time averaged autospectrum of sensors $A$

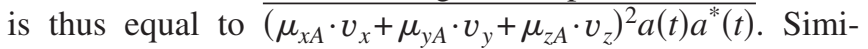
larly the time averaged cross spectrum of the signals of sensors $A$ and $B$, the latter with sensitivity unit vector $\boldsymbol{\mu}_{B}$ $=\left[\mu_{x B}, \mu_{y B}, \mu_{z B}\right]$ is equal to

$$
\overline{\left(\boldsymbol{\mu}_{A} \cdot \mathbf{v}\right)\left(\boldsymbol{\mu}_{B} \cdot \mathbf{v}\right) a(t) a^{*}(t)}=\overline{\left(\mu_{x A} \cdot v_{x}+\mu_{y A} \cdot v_{y}+\mu_{z A} \cdot v_{z}\right)\left(\mu_{x B} \cdot v_{x}+\mu_{y B} \cdot v_{y}+\mu_{z B} \cdot v_{z}\right) a(t) a^{*}(t)}
$$

In the auto- and cross-spectrum the same term $a(t) a^{*}(t)$ appears, and in a comparison between these spectra this term can thus be omitted. For the four $v$ sensors, as defined above, the four autospectra and the six cross spectra are

$$
\begin{aligned}
& A_{1}=v_{x}^{2} / 2+v_{z}^{2} / 2+v_{x} v_{z}+v_{\mathrm{rev}}^{2} / 3, \\
& A_{2}=v_{y}^{2} / 2+v_{z}^{2} / 2+v_{y} v_{z}+v_{\mathrm{rev}}^{2} / 3, \\
& A_{3}=v_{x}^{2} / 2+v_{z}^{2} / 2-v_{x} v_{z}+v_{\mathrm{rev}}^{2} / 3, \\
& A_{4}=v_{y}^{2} / 2+v_{z}^{2} / 2-v_{y} v_{z}+v_{\mathrm{rev}}^{2} / 3, \\
& C_{12}=v_{z}^{2} / 2+v_{x} v_{y} / 2+v_{x} v_{z} / 2+v_{y} v_{z} / 2+v_{\mathrm{rev}}^{2} / 6, \\
& C_{13}=-v_{x}^{2} / 2+v_{z}^{2} / 2,
\end{aligned}
$$

$$
\begin{aligned}
& C_{14}=v_{z}^{2} / 2-v_{x} v_{y} / 2+v_{x} v_{z} / 2-v_{y} v_{z} / 2+v_{\mathrm{rev}}^{2} / 6, \\
& C_{23}=v_{z}^{2} / 2-v_{x} v_{y} / 2-v_{x} v_{z} / 2+v_{y} v_{z} / 2+v_{\mathrm{rev}}^{2} / 6, \\
& C_{24}=-v_{y}^{2} / 2+v_{z}^{2} / 2, \\
& C_{34}=v_{z}^{2} / 2+v_{x} v_{y} / 2-v_{x} v_{z} / 2-v_{y} v_{z} / 2+v_{\mathrm{rev}}^{2} / 6 .
\end{aligned}
$$

In an experiment one thus determines ten spectra and there are four unknown quantities, $v_{x}, v_{y}, v_{z}$, and $v_{\text {rev }}$. As an approximation for the best solution of Eqs. (3)-(12) the following algebraic procedure was used. From Eqs. (3)-(12) the products of $v_{i} \cdot v_{j}$ are estimated

$$
v_{x} \cdot v_{y}=\left\{C_{12}-C_{14}-C_{23}+C_{34}\right\} / 2=C_{x y},
$$




$$
\begin{aligned}
& v_{x} \cdot v_{z}=\left\{A_{1}-A_{3}+C_{12}+C_{14}-C_{23}-C_{34}\right\} / 4=C_{x z}, \\
& v_{y} \cdot v_{z}=\left\{A_{2}-A_{4}+C_{12}-C_{14}+C_{23}-C_{34}\right\} / 4=C_{y z} .
\end{aligned}
$$

Use as variables $u=v_{x}^{2}, v=v_{y}^{2}$, and $w=v_{z}^{2}$, then one obtains the six following equations:

$$
\begin{aligned}
& u-v=A_{1}-A_{2}+A_{3}-A_{4}=A_{0}, \\
& -u+w=2 C_{13}, \\
& -v+w=2 C_{24}, \\
& u=\left(v_{x} \cdot v_{y}\right)\left(v_{x} \cdot v_{z}\right) /\left(v_{y} \cdot v_{z}\right)=C_{x y} \cdot C_{x z} / C_{y z}=C_{u}, \\
& v=\left(v_{x} \cdot v_{y}\right)\left(v_{y} \cdot v_{z}\right) /\left(v_{x} \cdot v_{z}\right)=C_{x y} \cdot C_{y z} / C_{x z}=C_{v}, \\
& w=\left(v_{x} \cdot v_{z}\right)\left(v_{y} \cdot v_{z}\right) /\left(v_{x} \cdot v_{y}\right)=C_{y z} \cdot C_{x z} / C_{x y}=C_{w} .
\end{aligned}
$$

These six equations with three unknown variables are solved using the least mean squares method; the expression

$$
\begin{aligned}
& \left(u-v-A_{0}\right)^{2}+\left(-u+w-2 C_{13}\right)^{2}+\left(-v+w-2 C_{24}\right)^{2} \\
& +\left(u-C_{u}\right)^{2}+\left(v-C_{v}\right)^{2}+\left(w-C_{w}\right)^{2},
\end{aligned}
$$

should have a minimum value. Differentiating with respect to the three variables gives

$$
\begin{array}{ll}
\partial / \partial u=0: & 3 u-v-w-A_{0}+2 C_{13}-C_{u}=0, \\
\partial / \partial v=0: & -u+3 v-w+A_{0}+2 C_{24}-C_{v}=0, \\
\partial / \partial w=0: & -u-v+3 w-2 C_{13}-2 C_{24}-C_{w}=0 .
\end{array}
$$

Solving these equations gives as the best solutions

$$
\begin{aligned}
& 4 u=4 v_{x}^{2}=A_{0}-2 C_{13}+2 C_{u}+C_{v}+C_{w}, \\
& 4 v=4 v_{y}^{2}=-A_{0}-2 C_{24}+C_{u}+2 C_{v}+C_{w}, \\
& 4 w=4 v_{z}^{2}=2 C_{13}+2 C_{24}+C_{u}+C_{v}+2 C_{w},
\end{aligned}
$$

from which the free field particle velocity vector and the reverberant field can be solved.

If the SNR (signal-to-noise ratio) in $A_{1}-A_{4}$ is low, but high in $C_{i j}$ then the autospectra should not be used in Eqs. (14) and (15).

An alternative and practical method to obtain the best solution of Eqs. (3)-(12) is to use a simple straightforward numerical procedure (on a PC) to find the minimum value of the expression

$$
\begin{aligned}
& \left(v_{x}^{2} / 2+v_{z}^{2} / 2+v_{x} v_{z}+v_{\mathrm{rev}}^{2} / 3-A_{1}\right)^{2}+\left(v_{y}^{2} / 2+v_{z}^{2} / 2+v_{y} v_{z}\right. \\
& \left.+v_{\mathrm{rev}}^{2} / 3-A_{2}\right)^{2}+\left(v_{x}^{2} / 2+v_{z}^{2} / 2-v_{x} v_{z}+v_{\mathrm{rev}}^{2} / 3-A_{3}\right)^{2} \\
& +\left(v_{y}^{2} / 2+v_{z}^{2} / 2-v_{y} v_{z}+v_{\mathrm{rev}}^{2} / 3-A_{4}\right)^{2}+\left(v_{z}^{2} / 2+v_{x} v_{y} / 2\right. \\
& \left.+v_{x} v_{z} / 2+v_{y} v_{z} / 2+v_{\mathrm{rev}}^{2} / 6-C_{12}\right)^{2}+\left(-v_{x}^{2} / 2+v_{z}^{2} / 2-C_{13}\right)^{2} \\
& +\left(v_{z}^{2} / 2-v_{x} v_{y} / 2+v_{x} v_{z} / 2-v_{y} v_{z} / 2+v_{\mathrm{rev}}^{2} / 6-C_{14}\right)^{2} \\
& +\left(v_{z}^{2} / 2-v_{x} v_{y} / 2-v_{x} v_{z} / 2+v_{y} v_{z} / 2+v_{\mathrm{rev}}^{2} / 6-C_{23}\right)^{2} \\
& +\left(-v_{y}^{2} / 2+v_{z}^{2} / 2-C_{24}\right)^{2}+\left(v_{z}^{2} / 2+v_{x} v_{y} / 2-v_{x} v_{z} / 2\right.
\end{aligned}
$$

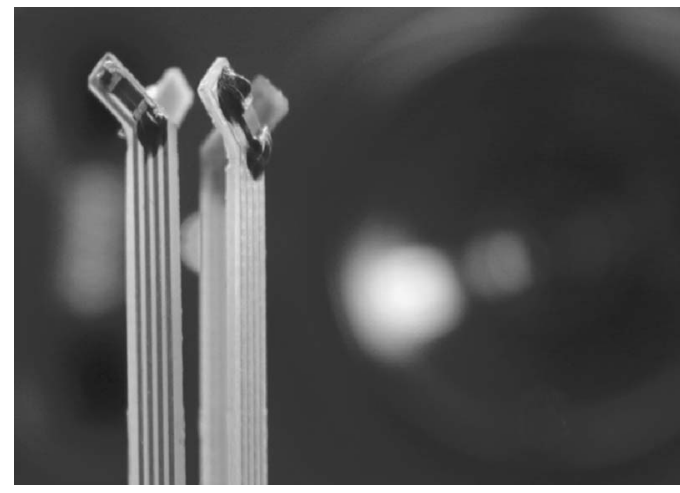

FIG. 3. The four sensor device, microflown elements bonded to an epoxy carrier.

$$
\left.-v_{y} v_{z} / 2+v_{\mathrm{rev}}^{2} / 6-C_{34}\right)^{2} .
$$

The values of $v_{x}, v_{y}$, and $v_{z}$, as found from Eqs. (25)-(27) and $v_{\text {rev }}^{2} \geqslant 0$ can be used as starting values in this numerical procedure.

\section{THE FOUR SENSOR DEVICE}

A photograph of the four $v$ sensor device is shown in Fig. 3. Four small printed circuit boards, each with one $\mu$ flown on it are combined. The orientations of the four $v$ sensors are $\boldsymbol{\mu}_{1}=[1,0,1] / \sqrt{ } 2, \quad \boldsymbol{\mu}_{2}=[0,1,1] / \sqrt{ } 2, \quad \boldsymbol{\mu}_{3}$ $=[-1,0,1] / \sqrt{ } 2$, and $\boldsymbol{\mu}_{4}=[0,-1,1] / \sqrt{ } 2$. For the configuration, as shown in Fig. 3 the directional characteristics of each sensor corresponds to $\cos \left(\alpha_{\mu \mathrm{v}}\right)$, see Fig. 4. It is necessary that the encapsulation is not done by combining the four printed circuit boards on a fixed bar, since this will lead to (frequency dependent) deviations of the directional characteristics as given in $\operatorname{Sec}$. I as $\cos \left(\alpha_{\mu \mathrm{v}}\right)$. The distance between the four sensors is only $5 \mathrm{~mm}$. The main electronics are encapsulated in the holder and a seven-pin connector is used for the power supply and connection to a sound card or analyzer.

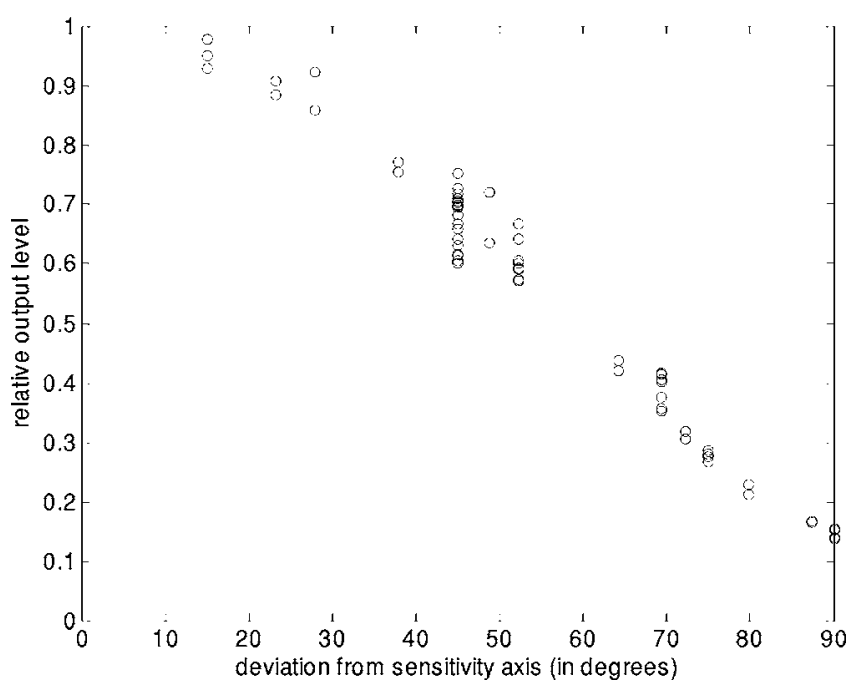

FIG. 4. Directionality measurement of a microflown element, relative sensitivity against deviation from maximum sensitivity direction. 


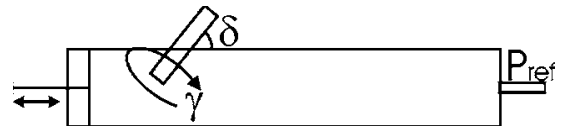

FIG. 5. Standing wave tube, a calibration device.

The calibration of the $v$ sensors has been described extensively in a number of papers. ${ }^{3,4,6}$ There are different methods to obtain a calibrated $v$ sensor; three methods are mentioned below.

1. A calibrated $p$ sensor and the $v$ sensor in a standing wave tube and using the relation between the position dependence of the pressure and particle velocity.

2. A calibrated $p$ sensor and a $v$ sensor in a free field and far field situation, where $v=p /(\rho c)$.

3. Measure the particle velocity using the Laser Doppler Anemometry (LDA) method, ${ }^{4}$ and compare it with the signal from the $v$ sensor. The LDA is an independent method to measure the particle velocity. ${ }^{4}$

A practical method is to use a one-dimensional standing wave tube; a schematic drawing is shown in Fig. 5. The method to obtain the sensitivity of the $v$ sensor as a function of frequency from measurements in a standing wave tube (SWT), has been described by Raangs et al. ${ }^{4}$ The microphone is positioned at the end of the tube $(x=1)$, the $v$ sensor at a position $x$, where $v(x)$ is measured. A relation between $v(x)$ and $p(x=1)$ is used, where the damping is taken into account in a standing wave ratio (SWR)

$$
\begin{aligned}
\frac{v(x)}{p(l)}= & \frac{1}{\rho_{0} c}\left[\frac{1}{S W R} \cos \{k(\ell-x)-\vartheta / 2\}+j \sin \{k(l-x)\right. \\
& -\vartheta / 2\}],
\end{aligned}
$$

with $\vartheta$ a phase angle.

In the case where the damping is neglected, a simple relation remains. In our experiments the damping was quite small, a SWR of about $20-25 \mathrm{~dB}$ was measured. The measured data are corrected using Eq. (29) and are then fitted with a good approximation of a Microflown ${ }^{11}$ output $=\frac{L F S}{\sqrt{\left(1+f^{2} / f_{\text {hea cap cap }}^{\text {re }}\left(1+f^{2} / f_{\text {diff }}^{2}\right.\right.}}$, with LFS the low frequency sensitivity, $f_{\text {diff }}$ the diffusion corner frequency, and $f_{\text {heat cap }}$ the heat capacity corner frequency. It should be noticed that for interpretation of our experiments the differences between the sensitivities are of importance, and not the absolute sensitivity; systematic errors in the sensitivities of the four sensors do not play a role.

Simple relations can be derived for the position dependence and the relation between pressure and particle velocity. A number of $p$ and $v$ sensors can therefore be placed at different positions in the standing wave tube. In Fig. 5 the (not calibrated) $v$ sensor is positioned under an angle $\delta$ with the standing wave tube, together with calibrated, reference, $p$ and $v$ sensors.

The four $v$ sensors (and the $x-y$ axes) can be rotated around the probe holder, which is defined as the $z$ axis; the rotation angle $\gamma=0$ corresponds with the drawing in Fig. 5,

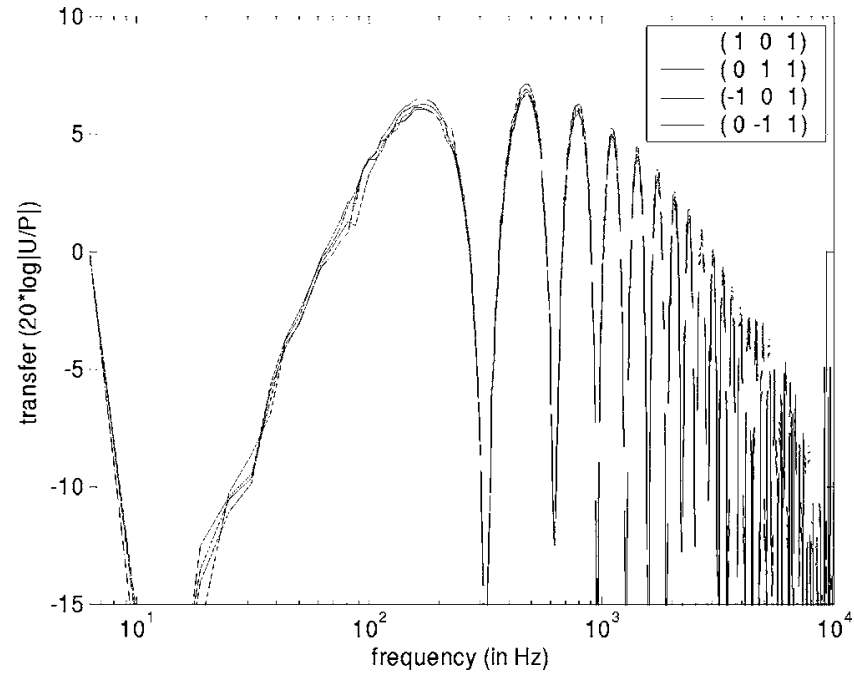

FIG. 6. Transfer function between microflown and reference microphone $(14 \mathrm{mV} / \mathrm{Pa})$ in a standing wave tube. Calibration values can be obtained at the maxima. (Differences in sensitivity are approximately $1 / 2 \mathrm{~dB}$.)

i.e., the $x$ axis in the plane of the drawing and the $y$ axis perpendicular to it. Due to the rotation around the $z$ axis the particle velocity in the standing wave tube is written in this Cartesian coordinate system as: $\mathbf{v}$ $\propto[\cos (\delta) \cos (\gamma), \cos (\delta) \sin (\gamma), \sin (\delta)]$; the four unit vectors of the $v$ sensors remain constant and equal to the values given above. The directional characteristics of for instance $v$ sensor one thus become $\mathbf{v} \cdot \mathbf{n}_{1} \propto \cos (\delta) \cos (\gamma)+\sin (\delta)$ and of $v$ sensor two $\mathbf{v} \cdot \mathbf{n}_{2} \propto \cos (\delta) \sin (\gamma)+\sin (\delta)$.

In Fig. 6 the measured output in the $\boldsymbol{\mu}_{\mathrm{i}}$ direction as a function of frequency is shown for the four $v$ sensors. The curves show that the differences between the four $v$ sensors are quite small.

\section{EXPERIMENTS IN A REVERBERANT ROOM}

In a reverberant room with dimensions of about $9^{*} 7^{*} 4 \mathrm{~m}$ a loudspeaker box with dimensions $0.4^{*} 0.2^{*} 0.2 \mathrm{~m}$ was used as a sound source. The four $v$-sensor device was placed at various locations in the room, in such a way that the inclination (angle $\delta$ with the probe holder) and rotation angle (angle $\gamma$ in a plane perpendicular to the probe holder) were varied. The source-sensor distance was also varied in order to create a situation where $v_{\text {direct }}^{2} / v_{\text {reverberant }}^{2}<1$ and $v_{\text {direct }}^{2} / v_{\text {reverberant }}^{2}>1$. When the inclination angle was taken as $\delta$, the rotation angle as $\gamma$ and the sound source-sensor distance as $r$, the response was measured for $r=0.5,1$, and $2 \mathrm{~m}$, $\delta$ as well as $\gamma=0,30,60$, and $90 \mathrm{deg}$; so in total 48 responses. As the signal to the loudspeaker white noise was used; the same (white noise) time signal was used in the 48 experiments. The auto- and cross-spectra were averaged over $4 \mathrm{~s}$.

A four input channel device (product name Siglab) and a sound card in a PC were used as input for calculating the auto- and cross-spectra. 
TABLE I. Results for one meter distance to the source, the bold numbers are actual angles, the measured angles are given in the table.

\begin{tabular}{ccccc}
\hline \hline Incl, $\delta \downarrow \backslash$ Rot, $\gamma$ & $\mathbf{0}$ & $\mathbf{3 0}$ & $\mathbf{6 0}$ & $\mathbf{9 0}$ \\
\hline $\mathbf{0}$ & $-0.79 \backslash 1.2$ & $1.47 \backslash 31$ & $0.93 \backslash 61.1$ & $0.74 \backslash 89.6$ \\
$\mathbf{3 0}$ & $29.1 \backslash 1.1$ & $29.0 \backslash 28.3$ & $29.4 \backslash 57.8$ & $28.2 \backslash 86.6$ \\
$\mathbf{6 0}$ & $57.4 \backslash-1.9$ & $57.0 \backslash 29.8$ & $57.6 \backslash 59.5$ & $56.1 \backslash 91.1$ \\
$\mathbf{9 0}$ & $87.7 \backslash(17)$ & $86.0 \backslash(-7)$ & $86.7 \backslash(-9)$ & $85.9 \backslash(-30)$ \\
\hline \hline
\end{tabular}

\section{RESULTS}

In acoustic measurements it may well be that one is primarily interested in the free field properties of a sound source; on the other hand there are situations where one is primarily interested in the properties of the reverberant sound field. An example of the first case is that the one wants to know the radiated power (noise) of a machine while it is placed in a reverberant room. An example of the second case is that one is interested in the quality of a concert hall where the properties of the reverberant field are of interest. When using a $p$ sensor it is quite difficult or even impossible to measure the free and the reverberant field separately. When the distance $r$, between sound source and sensor is larger than the reverberation distance $r_{r}$, of the room it is difficult to measure accurately the free field on the other hand, when the distance is small it is difficult to determine the properties of the reverberant field (the reverberation distance $r_{r}$ is defined as the distance from the source where the rms value of the free field is equal to the rms value of the reverberant field). However with the 4- $v$-sensor device a number of possibilities appear. In Sec. VII A, the emphasis is on the determination of the free field, in Sec. VII B, the determination of the reverberant field is discussed. In the next two sections it will be shown that even in the case of $r>r_{r}$ it is possible to determine the free field, while for the case of $r<r_{r}$ the properties of the reverberant field can be determined.

\section{A. Free field, localization of the sound source}

The free or direct sound field is calculated from the measured ten auto- and cross-spectra as was explained in Sec. III. From the ratios of $v_{x} / v_{y}, v_{x} / v_{z}$, and $v_{y} / v_{z}$, of the direct field the direction of the sound source seen from the sensor is calculated and compared with the theoretical value as deduced from the experimental set up.

In Table I results for the case $r=1 \mathrm{~m}$ are given; the bold numbers refer to the angles in degrees of the experimental setup, the "theoretical angles." The other numbers for the angles in degrees are the results of the calculations from the experimental results. In, e.g., $29.1 \backslash 1.1$ the value of 29.1 refers to the value of $\delta$, and 1.1. to the value of $\gamma$.

The (numerical) calculated values for $v_{x}$ and $v_{y}$ for the case $\delta=90$ have no meaning and are placed between brackets. When $\delta=90, v_{x}=v_{y}=0$ and a value of $\arctan \left(v_{x} / v_{y}\right)$ is undefined. In practice such a situation is recognized when the solutions for the components of the vector $v$ are such that $v_{x} \ll v_{z}$ and $v_{y} \ll v_{z}$ while $v_{x}$ and $v_{y}$ are of the same order.

Of course the numerical procedure gives values for $v_{x}$ and $v_{y}$, but the ratio in $\arctan \left(v_{x} / v_{y}\right)$ has no meaning here.
TABLE II. Measurement and errors, $\beta$ is the deviation in degrees averaged over 16 measurements.

\begin{tabular}{ccccccc}
\hline \hline $\begin{array}{c}r \\
(\mathrm{~m})\end{array}$ & $v_{\mathrm{dir}}^{2} / v_{\mathrm{rev}}^{2}$ & $\cos (\beta)$ & $\begin{array}{c}\beta \\
(\mathrm{deg})\end{array}$ & $\sin (\beta)$ & $\sigma$ & $\begin{array}{c}\beta \\
(\mathrm{deg})\end{array}$ \\
\hline 0.5 & 13.2 & 0.9984 & 3.3 & 0.0513 & 0.025 & 3 \\
1 & 3.15 & 0.9989 & 2.7 & 0.0435 & 0.017 & 2.5 \\
2 & 0.72 & 0.9900 & 8 & 0.1322 & 0.049 & 7.5 \\
\hline \hline
\end{tabular}

The average reverberant sound field $v_{\text {rev }}^{2}$ is also calculated from these experimental values. In the second column of Table II an averaged value of the ratio direct/reverberant particle velocity, $v_{\mathrm{dir}}^{2} / v_{\text {rev }}^{2}$ is given. For a more detailed comparison between experimental and theoretical directions the inner, scalar, vector product of the theoretical particle velocity vector, taken as unit vector and denoted as $\mathbf{v}_{\text {th }}$, and the experimental particle velocity vector, denoted as $\mathbf{v}_{\text {exp }}$, is calculated. The deviation between the directions of $\mathbf{v}_{\text {th }}$ and $\mathbf{v}_{\text {exp }}$, denoted as $\beta$ is then found from: $\cos (\beta)=\left|\mathbf{v}_{\mathrm{th}} \cdot \mathbf{v}_{\text {exp }}\right| /\left|\mathbf{v}_{\text {exp }}\right|$. This deviation angle $\beta$ can also be found from the inner vector product as: $\sin (\beta)=\left|\mathbf{v}_{\text {th }} \boldsymbol{x} \mathbf{v}_{\text {exp }}\right| /\left|\mathbf{v}_{\text {exp }}\right|$, where the symbol $\boldsymbol{x}$ is used for the vector product. In terms of $\delta$ and $\gamma$ the theoretical particle velocity vector is written as $\mathbf{v}_{\text {th }}$ $=\mathrm{i}[\cos (\delta) \cos (\gamma)]+\mathrm{j}[\cos (\delta) \sin (\gamma)]+\mathrm{k}[\sin (\delta)]$. In the third and fifth columns of Table II, the averaged values of $\cos (\beta)$ and $\sin (\beta)$ are given, the averaging is done over the 16 different directions given by $\delta$ and $\gamma$. For the deviation expressed by $\sin (\beta)$ also a standard deviation $\sigma$ is given, calculated from: $\sigma=\sqrt{\operatorname{var}}=\frac{1}{n} \sqrt{\sum(\text { value }- \text { mean })^{2}}$, with $n=16$. The table shows a fairly good similarity between experimental found direction of the source and the real direction. So it is thus possible to measure the direct sound field in a reverberant environment using this 4- $v$-sensor device. Even for the case where the sound source-sensor distance is larger than the reverberation distance the results are within $8 \mathrm{deg}$.

\section{B. The reverberant field}

The fact that in the autospectrum of a $v$ sensor only $v_{\mathrm{rev}}^{2} / 3$ is measured means that only a part of the reverberant field contributes to it. Thus by measuring a number of autospectra (or cross spectra) information about different parts of the reverberant field can be obtained. For example, if there are three $v$ sensors, with $\boldsymbol{\mu}_{1}=[1,0,0], \boldsymbol{\mu}_{2}=[0,1,0]$, and $\boldsymbol{\mu}_{3}$ $=[0,0,1]$, then the signal of sensor one will contain the reverberant field around the $x$ axis, sensor two the field around the $y$ axis, and sensor three the field around the $z$ axis.

When the free field is known, it is in principle possible to calculate the reverberant field from the difference of the autospectrum and the free field. However, this will not be a good method when $r<r_{r}$ since the reverberant field is smaller than the free field; consider, e.g., the situation with $r=0.5 \mathrm{~m}$ in Table II, where $v_{\mathrm{dir}}^{2} / v_{\mathrm{rev}}^{2}=13.2$. It seems better to calculate it from experimental values, which, in the case of no reflections, should be zero. An example is the expression $A_{i} \cdot A_{j}-C_{i j}^{2}$. In the case of no reflections $A_{i}=\left(\mathbf{v} \cdot \boldsymbol{\mu}_{i}\right)^{2}$, $A_{j}=\left(\mathbf{v} \cdot \boldsymbol{\mu}_{j}\right)^{2}$, and $C_{i j}=\left(A_{i} \cdot A_{j}\right)=\left(\mathbf{v} \cdot \boldsymbol{\mu}_{i}\right)\left(\mathbf{v} \cdot \boldsymbol{\mu}_{j}\right)$ thus $A_{i} \cdot A_{j}-C_{i j}^{2}$ vanishes. In the case of reflections $A_{i} \cdot A_{j}-C_{i j}^{2}$ can be written as a quadratic expression of $v_{\mathrm{rev}}^{2}$, which then can be solved; 
TABLE III. Reverberant field $v_{\text {rev }}^{2}$, calculated from two sensors at the time, values were normalized with respect to $v_{\mathrm{dir}}^{2}$ at $r=1 \mathrm{~m}$.

\begin{tabular}{ccccccc}
\hline \hline$r \backslash \gamma \backslash \delta$ & $v_{\text {rev }}^{2}(1,2)$ & $v_{\text {rev }}^{2}(1,3)$ & $v_{\text {rev }}^{2}(1,4)$ & $v_{\text {rev }}^{2}(2,3)$ & $v_{\text {rev }}^{2}(2,4)$ & $v_{\text {rev }}^{2}(3,4)$ \\
\hline $0.5 \backslash 60 \backslash 0$ & 0.400 & 0.413 & 0.360 & 0.280 & 0.320 & 0.288 \\
$0.5 \backslash 90 \backslash 0$ & 0.331 & 0.251 & 0.332 & 0.266 & 0.422 & 0.265 \\
$0.5 \backslash 90 \backslash 30$ & 0.341 & 0.347 & 0.377 & 0.435 & 0.368 & 0.338 \\
$0.5 \backslash 0 \backslash 60$ & 0.398 & 0.406 & 0.325 & 0.404 & 0.301 & 0.379 \\
$1 \backslash 30 \backslash 0$ & 0.323 & 0.377 & 0.304 & 0.286 & 0.330 & 0.289 \\
$1 \backslash 60 \backslash 30$ & 0.331 & 0.274 & 0.317 & 0.274 & 0.318 & 0.265 \\
\hline \hline
\end{tabular}

the simple and straightforward algebra for the cases $i=1, j$ $=2$, and $i=1$ and $j=3$ is given in Appendix B. A reason for taking $A_{i} \cdot A_{j}-C_{i j}^{2}$ is that this quantity is not sensitive to deviations in the sensitivity or orientation vector $\boldsymbol{\mu}$ of the separate sensors, or to a (small) deviation in the orientation of the 4- $v$-sensor with respect to the desired experimental setup. However, the noise and SNR ratio can be different for $A_{i}$ and $C_{i j}$.

For the 4- $v$-sensor device there are six independent values of $A_{i} \cdot A_{j}-C_{i j}^{2}$, thus six different contributions of the reflections to the total reverberant field.

Some examples of results for $v_{\text {rev }}^{2}$ are given in Table III. In this table $r \backslash \gamma \backslash \delta=0.5 \backslash 60 \backslash 0$ refers to the experimental setup where the distance is $0.5 \mathrm{~m}$, the inclination $\delta=0$ and the angle $\gamma=60 \mathrm{deg} ; v_{\mathrm{rev}}^{2}(1,2)$ is the result using $A_{1} \cdot A_{2}$ $-C_{12}^{2}$. The values of $v_{\text {rev }}^{2}$ in Table III have been normalized with respect to the average value of $v_{\text {dir }}^{2}$ for $r=1 \mathrm{~m}$. This is done because a value of $v_{\text {rev }}^{2}$ in $(\mathrm{m} / \mathrm{s})^{2}$ does not give any information, unless the excitation used is known, together with the knowledge of a number quantities as, e.g., the loudspeaker efficiency, the radiation impedance, etc. A direct measure for comparison is the direct (or free) sound field $v_{\mathrm{dir}}^{2}$ (the choice of $r=1 \mathrm{~m}$ is of course arbitrary).

So by measuring and calculating the parts of the reverberant field information can be obtained about the contributions from different directions to the total reverberant sound field, which in turn gives information about the acoustics of the room.

In this paper, with emphasis on the 4-v-sensor device, we will not discuss these aspects.

In general one is inclined to think that the average reverberant sound field is independent of the distance $r$. For the three distances $r=0.5,1$, and $2 \mathrm{~m}$ the results are given in Table IV; in the second column the average value of $v_{\text {rev }}^{2}$, again normalized with respect to $v_{\mathrm{dir}}^{2}(r=1 \mathrm{~m})$, in the third column the standard deviation $\sigma$. The values of $v_{\mathrm{rev}}^{2}$ for the different distances are not exactly equal; however their differences are within the standard deviation.

TABLE IV. Reverberant field measured, normalized with respect to $v_{\text {dir }}^{2}(r$ $=1 \mathrm{~m})$, and standard deviation.

\begin{tabular}{ccc}
\hline \hline $\begin{array}{c}r \\
(\mathrm{~m})\end{array}$ & $v_{\mathrm{rev}}^{2}$ & $\sigma$ \\
\hline 0.5 & 0.358 & 0.066 \\
1 & 0.310 & 0.041 \\
2 & 0.299 & 0.074 \\
\hline
\end{tabular}

\section{CONCLUSIONS AND FUTURE PLANS}

A four-particle velocity sensor has been described with which the free field and the reverberant sound field can be detected in a reverberant environment. The strength of these sound fields is not expressed in terms of the sound pressure but in terms of the particle velocity. Since the latter is a vector, while the pressure is a scalar, more information can be obtained when using a particle velocity sensor instead of a pressure sensor (microphone). Free field measurements are desired for determining the radiated noise of a source, when it is positioned in a reverberant environment. In (concert) hall acoustics a precise and detailed knowledge of the reflection pattern and reverberant sound field is desired. From measurements in a reverberant room the free field sound field, as well as the reverberant sound have been deduced. This has been done for a source-sensor distance as well as smaller and larger than the reverberation distance of the room. Also information about the directional characteristics of the reverberant field is obtained by comparing the various auto and cross correlations of the four sensors.

The separate four $v$ sensors are quite small $\left(4^{*} 1.5^{*} 1.5 \mathrm{~mm}\right)$ and are encapsulated close to each other, so that the sensing volume (the volume in which the three vector components of the particle velocity are measured) is small as well; about $5^{*} 5^{*} 5 \mathrm{~mm}$.

Many separate $v$ sensors were processed on a wafer in a thin film technology. They were cut, glued on a small printed circuit boards with electrical contact areas. The four printed circuit boards were encapsulated together to one device. The next step in the integration process is to integrate all sensors on one die (a piece of silicon), a photo of this developed

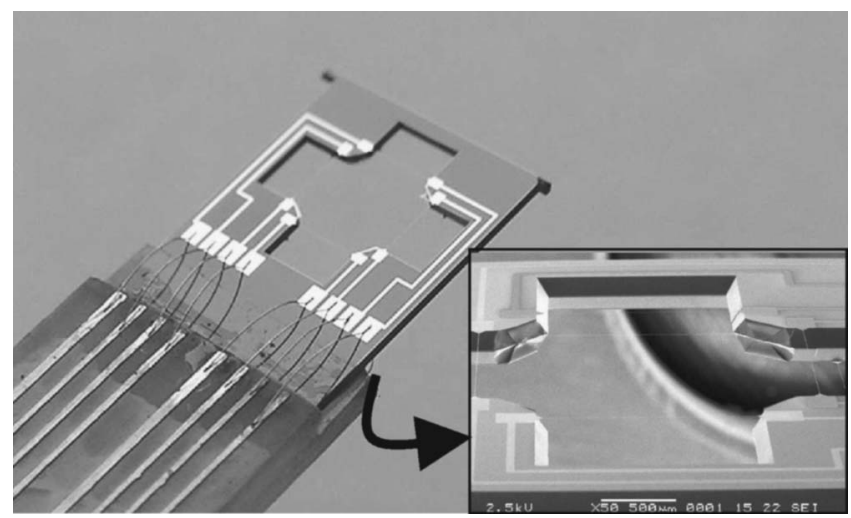

FIG. 7. Photo of the newly made device. The sensor wires can be seen inside the cross shape as thin lines. 
sensor is shown in Fig. 7. The mounting of the sensor is much simpler (only one printed circuit board is needed with electrical contacts), the directions of the four unit vectors $\boldsymbol{\mu}_{\mathbf{i}}$ are now precisely defined by a lithography process step, and also the equality of the four $v$ sensors in one device is even better.

\section{APPENDIX A}

$$
\begin{aligned}
\left(\boldsymbol{\mu}_{1} \cdot \mathbf{v}_{m}\right)\left(\boldsymbol{\mu}_{2} \cdot \mathbf{v}_{m}\right)= & v_{m}^{2}\left\{\mu_{1 x} \cdot \mu_{2 x} \cdot \sin ^{2}(\alpha) \cdot \cos ^{2}(\beta)\right. \\
& +\mu_{1 y} \cdot \mu_{2 y} \cdot \sin ^{2}(\alpha) \sin ^{2}(\beta) \\
& \left.+\mu_{1 z} \cdot \mu_{2 z} \cdot \cos ^{2}(\alpha)\right\} \\
& +v_{m}^{2}\left[\mu_{1 x} \cdot \mu_{2 y} \cdot \sin ^{2}(\alpha) \cos ^{2}(\beta) \sin (\beta)\right. \\
& +\mu_{1 x} \cdot \mu_{2 z} \cdot \sin (\alpha) \cos (\alpha) \cos (\beta) \\
& +\mu_{1 y} \cdot \mu_{2 x} \cdot \sin ^{2}(\alpha) \cos ^{2}(\beta) \sin (\beta) \\
& +\mu_{1 y} \cdot \mu_{2 z} \cdot \sin (\alpha) \cos (\alpha) \sin (\beta) \\
& +\mu_{1 z} \cdot \mu_{2 x} \cdot \sin (\alpha) \cos (\alpha) \cos (\beta) \\
& \left.+\mu_{1 z} \cdot \mu_{2 y} \cdot \sin (\alpha) \cos (\alpha) \sin (\beta)\right] .
\end{aligned}
$$

The integration $\int_{0}^{\pi} \sin (\alpha) d \alpha \int_{0}^{2 \pi} d \beta$ of the terms between [] results in a zero value (e.g., $\int_{0}^{2 \pi} \cos (\beta) \sin (\beta) d \beta=0$, etc.). The integration $\int_{0}^{\pi} \sin (\alpha) d \alpha \int_{0}^{2 \pi} d \beta$ of the terms between \{\} results in

$$
\begin{aligned}
& (4 \pi / 3) v_{m}^{2}\left\{\mu_{1 x} \cdot \mu_{2 x}+\mu_{1 y} \cdot \mu_{2 y}+\mu_{1 z} \cdot \mu_{2 z}\right\} \\
& \quad=v_{\text {rev }}^{2}\left(\boldsymbol{\mu}_{1} \cdot \boldsymbol{\mu}_{2}\right) / 3
\end{aligned}
$$

(e.g., $v_{m}^{2} \int_{0}^{\pi} \sin (\alpha) d \alpha \int_{0}^{2 \pi} \sin ^{2}(\alpha) \cdot \cos ^{2}(\beta) d \beta=v_{m}^{2} \pi \int_{0}^{\pi} \sin ^{3}(\alpha) d \alpha$ $=4 \pi v_{m}^{2} / 3=v_{\mathrm{rev}}^{2} / 3 \quad$ or $\quad v_{m}^{2} \int_{0}^{\pi} \sin (\alpha) d \alpha \int_{0}^{2 \pi} \cos ^{2}(\alpha) d \beta$ $\left.=-v_{m}^{2} 2 \pi \int_{0}^{\pi} \cos ^{2}(\alpha) d \cos (\alpha)=4 \pi v_{m}^{2} / 3=v_{\text {rev }}^{2} / 3\right)$.

Instead of writing out all the components of the arbitrary vectors $\boldsymbol{\mu}_{1}$ and $\boldsymbol{\mu}_{\boldsymbol{2}}$ a faster derivation of Eq. (2) is possible. Since the integration should be performed over all mirror sources equally distributed over the sphere the result of the integration is independent of any rotation of the Cartesian coordinate system. Rotate then the coordinate system such that $\boldsymbol{\mu}_{1}=[0,0,1]$ and $\boldsymbol{\mu}_{2}=\left[0, \sin \left(\alpha_{\mu}\right), \cos \left(\alpha_{\mu}\right)\right]$. The angle between $\boldsymbol{\mu}_{1}$ and $\boldsymbol{\mu}_{2}$ is $\alpha_{\mu}$ and the inner product $\boldsymbol{\mu}_{\mathbf{1}} \cdot \boldsymbol{\mu}_{\mathbf{2}}$ is equal to $\cos \left(\alpha_{\mu}\right)$. The integral equation for the contribution of the reverberant field to the cross spectrum becomes:

$$
\begin{aligned}
& v_{m}^{2} \int_{0}^{\pi} \sin (\alpha) \cdot d \alpha \cdot \int_{0}^{2 \pi} d \beta\{[\cos (\alpha)] \\
& \left.\times\left[\sin \left(\alpha_{\mu}\right) \sin (\alpha) \sin (\beta)+\cos \left(\alpha_{\mu}\right) \cos (\alpha)\right]\right\} \\
& =2 \pi v_{m}^{2} \int_{0}^{\pi} \sin (\alpha) \cos ^{2}(\alpha) \cos \left(\alpha_{\mu}\right) d \alpha=\left(4 \pi v_{m}^{2} / 3\right) \cos \left(\alpha_{\mu}\right) \\
& =\left(v_{\mathrm{rev}}^{2} / 3\right)\left(\boldsymbol{\mu}_{1} \cdot \boldsymbol{\mu}_{2}\right) .
\end{aligned}
$$

\section{APPENDIX B}

$$
\begin{aligned}
A_{1} \cdot A_{2}-\left(C_{12}\right)^{2}= & \left\{v_{x}^{2} / 2+v_{z}^{2} / 2+v_{x} \cdot v_{z}+v_{\mathrm{rev}}^{2} / 3\right\}\left\{v_{y}^{2} / 2\right. \\
& \left.+v_{z}^{2} / 2+v_{y} \cdot v_{z}+v_{\mathrm{rev}}^{2} / 3\right\}
\end{aligned}
$$

$$
\begin{aligned}
- & \left\{v_{z}^{2} / 2+v_{x} \cdot v_{y} / 2+v_{x} \cdot v_{z} / 2+v_{y} \cdot v_{z} / 2+v_{\mathrm{rev}}^{2} / 6\right\}^{2} \\
= & v_{x}^{2} \cdot v_{y}^{2} / 4+v_{x}^{2} \cdot v_{z}^{2} / 4+v_{x}^{2} \cdot v_{y} \cdot v_{z} / 2+\left(v_{\mathrm{rev}}^{2} / 3\right)\left(v_{x}^{2} / 2+v_{z}^{2} / 2\right. \\
& \left.+v_{x} \cdot v_{z}+A_{2}\right)+v_{y}^{2} \cdot v_{z}^{2} / 4+v_{z}^{4} / 4 \\
+ & v_{y} \cdot v_{z}^{3} / 2+v_{x} \cdot v_{y}^{2} \cdot v_{z} / 2+v_{x} \cdot v_{z}^{3} / 2+v_{x} \cdot v_{y} \cdot v_{z}^{2}-\left\{v_{z}^{4} / 4\right. \\
+ & v_{x}^{2} \cdot v_{y}^{2} / 4+v_{x}^{2} \cdot v_{z}^{2} / 4+v_{y}^{2} \cdot v_{z}^{2} / 4 \\
+ & \left(v_{\mathrm{rev}}^{2} / 6\right)^{2}+v_{x} \cdot v_{y} \cdot v_{z}^{2} / 2+v_{x} \cdot v_{z}^{3} / 2+v_{y} \cdot v_{z}^{3} / 2+\left(v_{\mathrm{rev}}^{2} / 3\right) \\
\times & \left(v_{z}^{2} / 2+v_{x} \cdot v_{y} / 2+v_{x} \cdot v_{z} / 2+v_{y} \cdot v_{z} / 2\right) \\
+ & v_{x}^{2} \cdot v_{y} \cdot v_{z} / 2+v_{x} \cdot v_{y}^{2} \cdot v_{z} / 2+v_{x} \cdot v_{y} \cdot v_{z}^{2} / 2 \\
= & \left(v_{\mathrm{rev}}^{2} / 3\right)\left(A_{1}+A_{2}-v_{\mathrm{rev}}^{2} / 3\right)-\left\{\left(v_{\mathrm{rev}}^{2} / 6\right)^{2}+\left(v_{\mathrm{rev}}^{2} / 3\right)\left(C_{12}\right)\right. \\
& \left.-v_{\mathrm{rev}}^{2} / 6\right\} \text { or } \\
3\left(v_{\mathrm{rev}}^{2} / 6\right)^{2}-2\left(v_{\mathrm{rev}}^{2} / 6\right)\left(A_{1}+A_{2}-C_{12}\right)+\left(A_{1} \cdot A_{2}-C_{12}^{2}\right) & =0,
\end{aligned}
$$

from which $v_{\text {rev }}^{2}$ can be solved.

$$
\begin{aligned}
& A_{1} \cdot A_{3}-\left(C_{13}\right)^{2}=\left\{v_{x}^{2} / 2+v_{z}^{2} / 2+v_{x} \cdot v_{z}+v_{\mathrm{rev}}^{2} / 3\right\} \cdot\left\{v_{x}^{2} / 2\right. \\
& \left.+v_{z}^{2} / 2-v_{x} \cdot v_{z}+v_{\mathrm{rev}}^{2} / 3\right\}- \\
& \left\{-v_{x}^{2} / 2+v_{z}^{2} / 2\right\}=v_{x}^{4} / 4+v_{x}^{2} \cdot v_{z}^{2} / 4-v_{x}^{3} \cdot v_{z} / 2+\left(v_{\mathrm{rev}}^{2} / 3\right) \\
& \times\left(v_{x}^{2} / 2+v_{z}^{2} / 2+v_{x} \cdot v_{z}+v_{x}^{2} / 2+v_{z}^{2} / 2\right. \\
& \left.-v_{x} \cdot v_{z}+v_{\mathrm{rev}}^{2} / 3\right)+ \\
& v_{x}^{2} \cdot v_{z}^{2} / 4+v_{z}^{4} / 4-v_{x} \cdot v_{z}^{3} / 2+v_{x}^{3} \cdot v_{z} / 2+v_{x} \cdot v_{z}^{3} / 2-v_{x}^{2} \cdot v_{z}^{2} \\
& -v_{x}^{4} / 4-v_{z}^{4} / 4+v_{x}^{2} \cdot v_{z}^{2} / 2= \\
& \left(v_{\text {rev }}^{2} / 3\right)\left(A_{1}+A_{3}-v_{\text {rev }}^{2} / 3\right) \text { or } \\
& 4\left(v_{\mathrm{rev}}^{2} / 6\right)^{2}-2\left(v_{\mathrm{rev}}^{2} / 6\right)\left(A_{1}+A_{3}\right)+\left(A_{1} \cdot A_{3}-C_{13}^{2}\right)=0,
\end{aligned}
$$

from which $v_{\text {rev }}$ can be solved.

An alternative way is as follows. Write $A_{1}=A_{1}+2 \cdot R$, where $\quad R=v_{\mathrm{rev}}^{2} / 6 ; \quad A 2=A_{2}+2 \cdot R, \quad A 3=A_{3}+2 \cdot R, \quad C_{12}$ $=\sqrt{ }\left(A_{1} \cdot A_{2}\right)+R$ and $C_{13}=\sqrt{ }\left(A_{1} \cdot A_{3}\right)$.

$$
\begin{aligned}
A_{1} \cdot A_{2}-C_{12}^{2} & =\left(A_{1}+2 R\right)\left(A_{2}+2 R\right)-\left[\sqrt{ }\left(A_{1} \cdot A_{2}\right)+R\right] 2 \\
& =2 R\left\{A_{1}+A_{2}-\sqrt{ }\left(A_{1} \cdot A_{2}\right)\right\}+3 R^{2} .
\end{aligned}
$$

$A_{1}+A_{2}=A_{1}+A_{2}-4 R$ and $\sqrt{ }\left(A_{1} \cdot A_{2}\right)=C_{12}-R$, thus

$$
\begin{aligned}
A_{1} \cdot A_{2}-C_{12}^{2}= & 2 R\left\{A_{1}+A_{2}-4 R-C_{12}+R\right\}+3 R^{2} \text { or } \\
& -3 R^{2}+2 R\left(A_{1}+A_{2}-C_{12}\right)=A_{1} \cdot A_{2}-C_{12}^{2}
\end{aligned}
$$

$A_{1} \cdot A_{3}-\left(C_{13}\right)^{2}=A_{1} \cdot A_{3}+2 R\left(A_{1}+A_{3}\right)+4 R 2-A_{1} \cdot A_{3}=2 R\left(A_{1}\right.$ $\left.+A_{3}-4 R\right)+4 R^{2}=-4 R 2+2 R\left(A_{1}+A_{3}\right)$, which are the same as the above equations.

${ }^{1}$ M. J. Berliner and J. F. Lindberg, ed., "Acoustic particle velocity sensors: Design, performance, and applications," AIP Conference Proceedings 368 (Woodbury, New York, 1995), ISBN 1-56396-549-6; K. Kim, T. Gabrielson, and G. C. Lauchle, "Development of an accelerometer-based underwater acoustic intensity sensor," J. Acoust. Soc. Am. 116, 3384-3392 
(2004).

${ }^{2}$ A. Nordby and O.-H. Bjor, Proceedings of Internoise (1984), p. $1107-$ 1109; O.-H. Bjor and H. J. Krystad, Proc. of the Autumn Conference 1982 (Anon. Institute of Acoustics, Edinburgh, 1982), pp. B7.1-B7.5.

${ }^{3} \mathrm{H}-\mathrm{E}$ de Bree, "An overview of Microflown Technologies," Acta. Acust. Acust. 89, 163-172 (2003); The Microflown, ISBN 9036515793; www.microflown.com/

${ }^{4}$ R. Raangs, T. Schlicke, and Richard Barham, "Calibration of a micromachined particle velocity microphone in a standing wave tube using a LDA photon correlation technique,” Meas. Sci. Technol.. 16, 1099-1108 (2005).

${ }^{5}$ V. B. Svetovoy and I. A. Winter, "Model of the $\mu$-flown microphone," Sens. Actuators 86, 171-181 (2000).

${ }^{6}$ J. W. van Honschoten, W. F. Druyvesteyn, H. Kuipers, R. Raangs, and G. J. M. Krijnen, "Selfnoise reduction in acoustic measurements with a particle velocity sensor by means of a cross correlation technique," Acta. Acust. 90, 349-355 (2004).
${ }^{7}$ H-E de Bree, R. Raangs, and W. F. Druyvesteyn, "Sound intensity measurements with the microflown sensor," Proc. Internoise 2004, Prague, session AN3, Paper 3, Fig. 3 (Microflown Technologies B.V., University of Twente, 2004).

${ }^{8}$ V. A. Gordienko, B. I. Goncharenko, and Ya. A. Ilyushin, "Basic rules of vector-phase structure formulation of the ocean noise field," Acoust. Phys. 39, 237-242 (1993).

${ }^{9}$ V. A. Shchurov, Modern State and Prospects for Use of Underwater Acoustic Intensity Measurements (Pacific Oceanological Institute, Vladivostok, 1998).

${ }^{10}$ F. J. Fahy, Sound Intensity, 2nd ed. (E \& FN Spon, London, 1995), ISBN 0419198105 .

${ }^{11}$ R. Raangs, W. F. Druyvesteyn, and H. E. de Bree, J. Audio Eng. Soc. 51, 344-357 (2003); J. W. van Honschoten, G. J. M. Krijnen, V. B. Svetovoy, H. E. de Bree, and M. C. Elwenspoek, J. Micromech. Microeng. 14, 1468-1477 (2004). 